\title{
Ground State Properties and Glueball Spectrum in SU(2) Yang-Mills Theory using Gauge Invariant Variables
}

\author{
Cécile Martin and Dominique Vautherin
}

\author{
Division de Physique Théorique*, \\ Institut de Physique Nucléaire, \\ F-91406, Orsay Cedex, France
}

\begin{abstract}
We describe a nonperturbative calculation of the spectrum of $\mathrm{SU}(2)$ Yang-Mills theory based on a Hamiltonian formulation. Our approach exploits gauge invariant variables similar to those used in nuclear physics to describe collective motion in nuclei.
\end{abstract}

IPNO/TH 93-68

*Unité de Recherche des Universités Paris XI et Paris VI associée au C.N.R.S 
Our understanding of the low energy behaviour of QCD depends crucially on the developpment of nonperturbative methods. The variational gaussian approximation which has been successfull in quantum mechanics and in scalar field theory remains difficult to apply for a nonabelian gauge theory because of the requirement to satisfy the Gauss law constraint. The necessity to maintain gauge invariance is however an essential ingredient in practical calculations. In order to study the configurations which contribute significantly to the nonperturbative ground state, we have to take into account the gauge invariant functional measure. The nontrivial gauge invariant volume element will induce a centrifugal effect and the boundary conditions on the wave functional will differ drastically from those in perturbative calculations. This has important phenomelogical consequences such as the occurence of nonvanishing condensates describing the vacuum state and the existence of a mass gap [回].

In this paper, we use a representation of the $\mathrm{SU}(2)$ vector potential which separates explicitly the gauge degrees of freedom [2, 3]. In this representation, the Gauss law appears as a local constraint. The hamiltonian however becomes non-local. A derivative expansion (or strong coupling expansion) valid in the nonperturbative domain and for slowly-varying fields allows one to write expliciltly the first few terms of an effective hamiltonian. One can thus obtain approximate solutions for the dynamics which are gauge invariant.

In section 2, we introduce more appropriate gauge invariant variables $\rho(x), \beta(x), \gamma(x)$. These variables describe the field configuration in an intrinsic frame and they can be interpreted as "density" and "deformation" variables. They are analogous to the collective variables introduced by Bohr and Mottelson to describe the dynamics of deformed nuclei «, 5, the pure gauge degree of freedom corresponding to intrinsic nucleon coordinates. The collective gauge invariant variables $\rho, \beta, \gamma$ allow one to describe deformed so- 
lutions of the dynamical equation without breaking the local gauge symmetry of the hamiltonian.

In section 3 and 4 , we work in the strong coupling limit which corresponds to the constant field approximation. We give the expression of the hamiltonian in terms of the gauge invariant variables $\rho, \beta, \gamma$. We investigate the properties of the ground state in the $\mathrm{SU}(2)$ gauge theory and we obtain predictions for the lowest masses of the color singlet bound states of gluons called glueballs. The minimum of the energy corresponds to a configuration with axial symmetry $(\gamma=0)$ and a strong deformation $(\beta$ near 1$)$. In this formalism, the lowest glueball states are interpreted as rotational levels and vibrational collective levels. In the last section, we discuss our results for the behaviour of the wavefunctional.

\section{The polar representation}

In the hamiltonian formalism, we choose the temporal gauge $A_{0}^{a}=0$. The hamiltonian reads :

$$
H=\frac{1}{2} \int d^{3} x \operatorname{tr}\left(E^{2}+B^{2}\right)
$$

For the $\mathrm{SU}(2)$ color group, the vector potential $A_{i a}$ (where $\mathrm{i}$ is a space index and $\mathrm{a}$ is a color index ) is a $3 \times 3$ matrix. The polar representation is given by [2, 3] :

$$
A_{i a}=f_{i n} \lambda_{n} h_{n a}-\frac{1}{2 g} h_{k b} \partial_{i} h_{k c} \epsilon_{a b c}
$$

where $\lambda_{n}, n=1,2,3$ are three numbers, $\hat{f}\left(\theta_{i}\right)$ and $\hat{h}\left(\phi_{a}\right)$ are orthogonal matrices parametrized by two sets of three Euler angles $\theta_{i}$ and $\phi_{a}$ and $g$ is the bare coupling constant. In terms of the $3 \times 3$ spin 1 matrices $\left(S_{i}\right)_{j k}=-i \epsilon_{i j k}$,

$$
\hat{f}=\exp \left(-i \theta_{1} S_{z}\right) \exp \left(-i \theta_{2} S_{y}\right) \exp \left(-i \theta_{3} S_{z}\right)
$$

The matrix $\hat{f}$ describes a rotation in ordinary space and the matrix $\hat{h}$ a rotation in color space. In general, $\lambda_{n}, \theta_{i}$ and $\phi_{a}$ are space-dependent. Under 
a local gauge transformation, the matrix $\hat{h}$ is simply rotated while $\hat{f}$ and $\lambda_{n}$ remain unchanged. Therefore, among the nine variables $A_{i a}$, six gauge invariant variables $\lambda_{n}$ and $\theta_{i}$ are explicitly separated from the three gauge degrees of freedom $\phi_{a}$.

In these new variables, the Gauss law operator

$$
G^{l}(x) \equiv \frac{1}{g} D_{i}^{l a} E_{i a},
$$

becomes a local operator :

$$
G^{l}(x)=J^{l}(x)
$$

where $J^{l}$ are the cartesian components of the color angular momentum in the laboratory frame expressed in terms of the Euler angles $\phi_{a}$ :

$$
J_{1}=-i\left\{-\cos \phi_{1} \cot \phi_{2} \frac{\partial}{\partial \phi_{1}}-\sin \phi_{1} \frac{\partial}{\partial \phi_{2}}+\frac{\cos \phi_{1}}{\sin \phi_{2}} \frac{\partial}{\partial \phi_{3}}\right\},
$$

with similar formulae for $J_{2}$ and $J_{3}$ [10]. For a color singlet state $|\Psi\rangle$

$$
G^{l}(x)\left|\Psi>=J^{l}(x)\right| \Psi>=0 .
$$

Therefore, the wave functional for a color singlet state depends only on the six gauge invariant variables : $\Psi\left(\lambda_{n}(x), \theta_{i}(x)\right)$. The functional integration measure becomes

$$
\prod_{i, a} D A_{i}^{a}(x)=\prod_{n>m}\left|\lambda_{n}^{2}(x)-\lambda_{m}^{2}(x)\right| \prod_{p} D \lambda_{p}(x) d \hat{f} d \hat{h},
$$

where $d \hat{f}=\sin \theta_{2} d \theta_{1} d \theta_{2} d \theta_{3}$, and $d \hat{h}=\sin \phi_{2} d \phi_{1} d \phi_{2} d \phi_{3}$.

In terms of the new gauge invariant variables, the hamiltonian becomes non local. The expression for the potential energy $\frac{1}{2} B^{2}$ is given by :

$$
V=\frac{g^{2}}{2}\left(\lambda_{1}^{2} \lambda_{2}^{2}+\lambda_{2}^{2} \lambda_{3}^{2}+\lambda_{1}^{2} \lambda_{3}^{2}\right)+\frac{1}{8} \sum_{n}\left(\operatorname{curl} \vec{w}_{n}\right)^{2},
$$

where $\vec{w}_{n}$ are the three orthogonal vectors : $w_{n}^{i}=f_{i n} \lambda_{n}$. 
The kinetic energy is a non local function of the gauge invariant quantities and their derivatives. However, we can use a derivative (or strong coupling) expansion to write explicitly the first few terms. This expansion will be valid in the nonperturbative domain and for slowly-varying fields. In the following, we will consider only the lowest order in $1 / g^{2}$, which corresponds also to the constant field approximation. At this order, we keep only the constant term in (2), which is the non-abelian term. This approach is therefore not adequate to describe the abelian limit.

For color singlet states, the kinetic energy is given by [2]

$$
\begin{aligned}
T= & -\frac{1}{2 L^{3}}\left\{\sum_{n=1}^{3} \frac{\delta^{2}}{\delta \lambda_{n} \delta \lambda_{n}}+2 \sum_{n>m} \frac{1}{\lambda_{n}^{2}-\lambda_{m}^{2}}\left(\lambda_{n} \frac{\delta}{\delta \lambda_{n}}-\lambda_{m} \frac{\delta}{\delta \lambda_{m}}\right)\right\} \\
& +\frac{1}{4 L^{3}} \sum_{k, n, m} \frac{\lambda_{n}^{2}+\lambda_{m}^{2}}{\left(\lambda_{n}^{2}-\lambda_{m}^{2}\right)^{2}} \epsilon_{n m k}^{2} L_{k}^{2},
\end{aligned}
$$

We have introduced a length scale $\mathrm{L}$, the total volume being $L^{3}$. The operators $L_{k}$ are the components of the angular momentum in the proper frame. As an example [10]:

$$
L_{x}=-i\left\{-\frac{\cos \theta_{3}}{\sin \theta_{2}} \frac{\partial}{\partial \theta_{1}}+\sin \theta_{3} \frac{\partial}{\partial \theta_{2}}+\cot \theta_{2} \cos \theta_{3} \frac{\partial}{\partial \theta_{3}}\right\} \text {. }
$$

In lowest order in $1 / g^{2}$, the angular momentum in the laboratory $M_{i}=f_{i k} L_{k}$ is equal to the spin density : $\vec{M}(x)=\vec{A}^{a}(x) \times \vec{E}^{a}(x)$.

It can be also usefull to write the expression of the gauge invariant operator $\vec{B}^{a} \cdot \vec{E}^{a}$. For color singlet states, it is given by :

$$
\vec{B}^{a} \cdot \vec{E}^{a}=-i g \epsilon_{n p q} \epsilon_{m p q} \lambda_{p} \lambda_{q}\left[\delta_{n m} \frac{\delta}{\delta \lambda_{m}}+i \frac{\epsilon_{k n m}}{\lambda_{n}^{2}-\lambda_{m}^{2}} \lambda_{m} L_{k}\right]
$$




\section{The gauge invariant collective coordinates $\rho(x), \beta(x), \gamma(x)$}

States of zero angular momentum depend only on three gauge invariant and rotational invariant quantities which can be choosen as [12] :

$$
\begin{aligned}
& B^{2}=B_{i}^{a}(x) B_{i}^{a}(x), \\
& B . y=B_{i}^{a}(x) y_{i}^{a}(x),
\end{aligned}
$$

and

$$
y^{2}=y_{i}^{a}(x) y_{i}^{a}(x)
$$

where $y_{i}^{a}(x)=\epsilon^{a b c} \epsilon_{i k l} B_{k}^{b}(x) B_{l}^{c}(x)$. For constant fields, they are related to the gauge invariant variables $\lambda_{n}$ according to the formulae :

$$
\begin{gathered}
B^{2}=g^{2}\left(\lambda_{1}^{2} \lambda_{2}^{2}+\lambda_{2}^{2} \lambda_{3}^{2}+\lambda_{1}^{2} \lambda_{3}^{2}\right), \\
y^{2}=4 g^{4} \lambda_{1}^{2} \lambda_{2}^{2} \lambda_{3}^{2}\left(\lambda_{1}^{2}+\lambda_{2}^{2}+\lambda_{3}^{2}\right), \\
B . y=6 g^{3} \lambda_{1}^{2} \lambda_{2}^{2} \lambda_{3}^{2} .
\end{gathered}
$$

These expresions, as well as the functional integration measure (8), are invariant under a permutation of the $\lambda_{n}$ and a simultaneous change of sign of two $\lambda_{n}$.

In the following, we will use three gauge invariant variables $\rho, \beta, \gamma$ defined as :

$$
\begin{gathered}
\rho^{2}=\lambda_{1}^{2}+\lambda_{2}^{2}+\lambda_{3}^{2}, \\
\lambda_{2}^{2}-\lambda_{1}^{2}=\frac{2}{\sqrt{3}} \rho^{2} \beta \sin \gamma, \\
2 \lambda_{3}^{2}-\left(\lambda_{1}^{2}+\lambda_{2}^{2}\right)=2 \rho^{2} \beta \cos \gamma,
\end{gathered}
$$

i.e.,

$$
\lambda_{1}^{2}-\frac{\rho^{2}}{3}=\frac{2}{3} \rho^{2} \beta \cos \left(\gamma+\frac{2 \pi}{3}\right) .
$$




$$
\begin{gathered}
\lambda_{2}^{2}-\frac{\rho^{2}}{3}=\frac{2}{3} \rho^{2} \beta \cos \left(\gamma-\frac{2 \pi}{3}\right) . \\
\lambda_{3}^{2}-\frac{\rho^{2}}{3}=\frac{2}{3} \rho^{2} \beta \cos \gamma .
\end{gathered}
$$

where $0<\beta<1$ and, from symmetry properties, we can restrict the angle $\gamma$ between 0 and $\pi / 3$. The variable $\rho$ has the dimension of $1 / L$.

The vibrational part of the wave function ( i. e. the part of the wave function which is independent of the three Euler angles $\theta_{i}$ ) is a function of the three gauge invariant collective coordinates $\rho, \beta, \gamma: \Psi(\rho, \beta, \gamma)$. The variables $\rho, \beta, \gamma$ are interpreted as the analogous of the density and deformation variables used in the collective model of Bohr in Nuclear Physics [4]. They remind also the derivation of the collective hamiltonian for a system of $\mathrm{N}$ particules by introducing $3 \mathrm{~N}-9$ Euler angles and six collective coordinates which describe the shape, the dimension and the orientation of the system [5]. Since we start from nine degrees of freedom $A_{i}^{a}$ for the Yang-Mills system, the description in terms of the gauge invariant variables corresponds to the treatment of the four-body problem [5]. The variables $\rho, \beta, \gamma$ will describe the gluon configuration in the intrinsic frame. The $\rho$ vibrations correspond to density vibrations of monopole character. The $\beta$ and $\gamma$ vibrations correspond to quadrupole oscillations. In general, there will be a coupling between the oscillations of the density $\rho$ and those of the deformations $\beta, \gamma$. We will show that $\rho, \beta$ and $\gamma$ are convenient coordinates to perform practical calculations. Furthermore, they give a physical insight in the structure of the vacuum state and the lowest excited states.

In the $\beta, \gamma$ plane, the axis $\beta=0$ corresponds to a "spherical" field configuration : $\lambda_{1}=\lambda_{2}=\lambda_{3}$. The axis $\gamma=0$ corresponds to an "axial symmetric" field configuration :

$$
\begin{gathered}
\lambda_{1}^{2}=\lambda_{2}^{2}=\frac{\rho^{2}}{3}\left(1-\beta^{2}\right), \\
\lambda_{3}^{2}=\frac{\rho^{2}}{3}(1+2 \beta) .
\end{gathered}
$$


The point $\beta=1, \gamma=0$ corresponds to two vanishing $\lambda$ 's : $\lambda_{1}=\lambda_{2}=0$, and $\lambda_{3}^{2}=\rho^{2}$. We point out that this configuration does not describe an abelian type field. Indeed, in the strong coupling approximation which corresponds to the constant potential approximation, we are not in a position to investigate the abelian limit.

An arbitrary point in the $\beta, \gamma$ plane corresponds to a "triaxial" field configuration.

The gauge invariant quantities in eq. (16-18) become functions of $\rho^{2}, \beta^{2}$ and $\beta^{3} \cos 3 \gamma$ :

$$
\begin{gathered}
\frac{B^{2}}{2}=g^{2} \frac{\rho^{4}}{6}\left(1-\beta^{2}\right), \\
B . y=g^{3} \frac{2}{3} \rho^{6}\left(\frac{1}{3}-\beta^{2}+\frac{2}{3} \beta^{3} \cos 3 \gamma\right),
\end{gathered}
$$

and the expression for the volume element of the vibrational coordinates

$$
d \tau=\left|\lambda_{1}^{2}-\lambda_{2}^{2}\right|\left|\lambda_{2}^{2}-\lambda_{3}^{2}\right|\left|\lambda_{1}^{2}-\lambda_{3}^{2}\right| d \lambda_{1} d \lambda_{2} d \lambda_{3}
$$

becomes :

$$
d \tau=\frac{2}{3 \sqrt{3}} \rho^{6} \beta^{3}|\sin 3 \gamma||\operatorname{det} M| d \rho d \beta d \gamma,
$$

where the jacobian is given by

$$
\operatorname{det} M \equiv \frac{D\left(\lambda_{1} \lambda_{2} \lambda_{3}\right)}{D(\rho \beta \gamma)}=\frac{1}{6 \sqrt{3} \lambda_{1} \lambda_{2} \lambda_{3}} \rho^{5} \beta
$$

A singular point occurs when one of the $\lambda_{n}$ vanishes or when two $\lambda$ 's are equal. This will imply suitable boundary conditions on the gauge invariant wave function $\Psi(\rho, \beta, \gamma)$. Let us now use the following rescaling :

$$
\Phi(\rho, \beta, \gamma)=\rho^{11 / 2} \frac{1}{\left|\lambda_{1} \lambda_{2} \lambda_{3}\right|^{1 / 2}} \Psi(\rho, \beta, \gamma) .
$$

The wave function $\Phi$ is normalized according to the integration measure

$$
\beta^{4}|\sin 3 \gamma| d \rho d \beta d \gamma
$$




\section{Strong coupling expansion}

In terms of the new gauge invariant variables $\rho, \beta, \gamma$ and after the rescaling (32), the vibrational hamiltonian (i.e. the terms in equations (9) and (10) independent of the angular momentum operators $L_{k}$ ) is equal to $T_{v i b}+V^{\prime}$ with

$$
\begin{aligned}
T_{v i b}=-\frac{1}{2 L^{3}} \quad & \left\{\frac{\partial^{2}}{\partial \rho^{2}}\right. \\
& +\frac{2}{\rho^{2}}\left(1+\beta \cos 3 \gamma-2 \beta^{2}\right) \frac{\partial^{2}}{\partial \beta^{2}}+\frac{2}{\rho^{2} \beta^{2}}(1-\beta \cos 3 \gamma) \frac{\partial^{2}}{\partial \gamma^{2}} \\
& -\frac{4}{\rho^{2}} \sin 3 \gamma \frac{\partial^{2}}{\partial \beta \partial \gamma}+\frac{2}{\rho^{2} \beta}\left(4-\beta \cos 3 \gamma-12 \beta^{2}\right) \frac{\partial}{\partial \beta} \\
& \left.+\frac{2}{\rho^{2} \beta^{2} \sin 3 \gamma}\left(3 \cos 3 \gamma-3 \beta+2 \beta \sin ^{2} 3 \gamma\right) \frac{\partial}{\partial \gamma}\right\},
\end{aligned}
$$

and

$$
V^{\prime}(\rho, \beta, \gamma)=L^{3} \frac{g^{2}}{6} \rho^{4}\left(1-\beta^{2}\right)+\frac{99}{8 L^{3} \rho^{2}}+\frac{3}{8 L^{3} \rho^{2}} \frac{1-\beta^{2}}{\frac{1}{3}-\beta^{2}+\frac{2}{3} \beta^{3} \cos 3 \gamma} .
$$

We wish to stress that the last two terms in the expression of the potential energy (35) and in particular the $\gamma$-dependence arise from the nontrivial gauge invariant factor in the integration measure (30). This potential diverges for $\beta=1, \gamma=0$. Therefore, the wave function has to vanish at this point. For $\gamma=0$ and $\beta=1-\epsilon$, we have, in the limit of small $\epsilon$ :

$$
\Psi(\rho, \beta, \gamma)=\sqrt{3} \frac{\epsilon^{1 / 2}}{\rho^{8}} \Phi(\rho, \beta, \gamma)
$$

In the limit of small deformations $\beta \ll 1$, the vibrational kinetic energy can be written as :

$$
\begin{aligned}
T_{v i b}=-\frac{1}{2 L^{3}} & \left\{\frac{\partial^{2}}{\partial \rho^{2}}\right. \\
& \left.+\frac{2}{\rho^{2}}\left[\frac{1}{\beta^{4}} \frac{\partial}{\partial \beta}\left(\beta^{4} \frac{\partial}{\partial \beta}\right)+\frac{1}{\beta^{2} \sin 3 \gamma} \frac{\partial}{\partial \gamma}\left(\sin 3 \gamma \frac{\partial}{\partial \gamma}\right)\right]\right\} .
\end{aligned}
$$

We thus obtain in this limit the expression of Bohr and Mottelson for the vibrational kinetic energy. 
The next step is to find the equilibrium shape by minimizing the potential energy $V^{\prime}(\rho, \beta, \gamma)$ with respect to $\rho, \beta, \gamma$. For this purpose, we have calculated the derivatives of the potential and the general expression for the stability matrix.

Spherical configurations $\left(\beta=0\right.$ and $\left.L^{2} \bar{\rho}_{0}^{2}=g^{-2 / 3}\left(\frac{3^{4}}{2}\right)^{1 / 3}\right)$ correspond to maxima with an energy equal to : $\bar{V}^{\prime}\left(\bar{\rho}_{0}, \beta_{0}, \gamma=0\right)=5.9 \mathrm{~g}^{2 / 3} / \mathrm{L}$.

We have found a minimum at $\gamma=0, \beta=\bar{\beta}, \rho=\bar{\rho}$, where $\bar{\beta}$ and $\bar{\rho}$ are solutions of the following equations :

$$
\begin{gathered}
L^{6} g^{2} \bar{\rho}^{6}=-\frac{9}{4\left(\frac{1}{3}-\bar{\beta}^{2}+\frac{2}{3} \bar{\beta}^{3}\right)}\left(1-\frac{\left(1-\bar{\beta}^{2}\right)(1-\bar{\beta})}{\frac{1}{3}-\bar{\beta}^{2}+\frac{2}{3} \bar{\beta}^{3}}\right), \\
L^{6} g^{2} \bar{\rho}^{6}=\frac{9}{8\left(1-\bar{\beta}^{2}\right)}\left(33+\frac{1-\bar{\beta}^{2}}{\frac{1}{3}-\bar{\beta}^{2}+\frac{2}{3} \bar{\beta}^{3}}\right) .
\end{gathered}
$$

The two functions given by the right hand sides of eq. (38) and of eq. (39) intersect at $\bar{\beta}$ very near 1 . By writing $\beta=1-\epsilon$ and by keeping the lowest order in $\epsilon$, we find at the minimum

$$
\bar{\epsilon}=\frac{2}{33} .
$$

and

$$
L^{2} \bar{\rho}^{2}=\left(\frac{3}{2}\right)^{2 / 3} \frac{1}{g^{2 / 3} \bar{\epsilon}^{2 / 3}}=\left(\frac{99}{4}\right)^{2 / 3} \frac{1}{g^{2 / 3}} .
$$

Therefore the minimum of $V^{\prime}$ corresponds to a strongly deformed field configuration with axial symmetry. We obtain :

$$
\bar{V}^{\prime}\left(\bar{\rho}^{2}, \beta=1-\bar{\epsilon}, \gamma=0\right)=g^{2 / 3} \frac{3}{2}\left({\frac{99^{2 / 3}}{4}}^{2}\right) / L .
$$

The corresponding value of the magnetic field is :

$$
B^{2}=\frac{2}{3} g^{2} \bar{\epsilon} \bar{\rho}^{4}
$$

or

$$
L^{4} B^{2}=\left(\frac{99}{4}\right)^{1 / 3} g^{2 / 3}=g L \bar{\rho} .
$$


This minimum associated to a nonvanishing value of the magnetic field cannot be described by a perturbative approach which constructs a state around $B^{2}=0$.

In lowest order in $\bar{\epsilon}$ or in $1 / \bar{\epsilon}$, the eigenvalues of the stability matrix at the minimum are

$$
\begin{gathered}
\omega_{\rho}^{2}=\frac{9}{2}\left(\left(\frac{3}{2}\right)^{2 / 3}-\frac{1}{2}\left(\frac{3}{2}\right)^{-1 / 3}\right) g^{4 / 3} \bar{\epsilon}^{1 / 3}, \\
\omega_{\beta}^{2}=\left(\frac{3}{2}\right)^{1 / 3} g^{2 / 3} \frac{1}{\bar{\epsilon}^{7 / 3}}, \\
\omega_{\gamma}^{2}=3\left(\frac{3}{2}\right)^{1 / 3} g^{2 / 3} \frac{1}{\bar{\epsilon}^{7 / 3}},
\end{gathered}
$$

where we have used dimensionless quantities. To this order in $\bar{\epsilon}$, the eigenvectors of the stability matrix are given by the directions of $\delta \rho, \delta \epsilon$ and $\delta \gamma \equiv \eta$. Therefore, the quadratic expansion of the potential energy arround the minimum yields :

$$
V^{\prime}(\rho, \beta, \gamma)=\bar{V}^{\prime}(\bar{\rho}, \bar{\epsilon}, 0)+\frac{1}{2} L^{3} \omega_{\rho}^{2}(\delta \rho)^{2}+\frac{1}{2} L \omega_{\beta}^{2}(\delta \epsilon)^{2}+\frac{1}{2} L \omega_{\gamma}^{2} \eta^{2} .
$$

\section{Solutions of the rotation-vibration hamil- tonian}

In analogy with the procedure used in nuclear physics, it will be more convenient to redefine the wave function according to :

$$
\varphi(\rho, \beta, \gamma)=|\sin 3 \gamma|^{1 / 2} \Phi(\rho, \beta, \gamma)=\rho^{11 / 2} \frac{|\sin 3 \gamma|^{1 / 2}}{\left|\lambda_{1} \lambda_{2} \lambda_{3}\right|^{1 / 2}} \Psi(\rho, \beta, \gamma) .
$$

After this rescaling, the differential operator $T_{v i b}$ is transformed into $\tilde{T}_{v i b}+$ $V_{\text {add }}$. Near the minimum, $\rho=\bar{\rho}+\delta \rho, \beta=1-\bar{\epsilon}-\delta \epsilon, \gamma=\eta$, we have :

$$
\tilde{T}_{v i b}=-\frac{1}{2 L^{3}}\left\{\frac{\partial^{2}}{\partial \rho^{2}}+\frac{6 \bar{\epsilon}}{\bar{\rho}^{2}} \frac{\partial^{2}}{\partial \epsilon^{2}}+\frac{6}{\bar{\rho}^{2}} \frac{\partial}{\partial \epsilon}+\frac{2 \bar{\epsilon}}{\bar{\rho}^{2}} \frac{\partial^{2}}{\partial \eta^{2}}\right\},
$$


and

$$
V_{a d d}=-\frac{1}{4 L^{3}} \frac{\bar{\epsilon}}{\bar{\rho}^{2} \eta^{2}} .
$$

In $\tilde{T}_{v i b}$, we have neglected terms proportional to $\eta \frac{\partial}{\partial \eta}$.

The rotational kinetic energy in eq. (10) can be written as :

$$
T_{\text {rot }}=\frac{1}{2 L^{3}}\left[\frac{L_{1}^{2}}{\mathcal{J}_{1}}+\frac{L_{2}^{2}}{\mathcal{J}_{2}}+\frac{L_{3}^{2}}{\mathcal{J}_{3}}\right] .
$$

The rotational and the vibrational parts of the energy are coupled to each other due to the $\rho, \beta, \gamma$ dependence of the moments of inertia:

$$
\begin{gathered}
\mathcal{J}_{1}=2 \rho^{2} \beta^{2} \frac{\sin ^{2}(\gamma+2 \pi / 3)}{1-\beta \cos (\gamma+2 \pi / 3)}, \\
\mathcal{J}_{2}=2 \rho^{2} \beta^{2} \frac{\sin ^{2}(\gamma+\pi / 3)}{1+\beta \cos (\gamma+\pi / 3)}, \\
\mathcal{J}_{3}=2 \rho^{2} \beta^{2} \frac{\sin ^{2} \gamma}{1-\beta \cos \gamma} .
\end{gathered}
$$

The collective hamiltonian in terms of the Euler angles and the variables $\rho, \beta, \gamma$ is given by

$$
H=T_{r o t}+\tilde{T}_{v i b}+V_{a d d}+V^{\prime}(\rho, \beta, \gamma) .
$$

The wave functions are normalized according to the volume element $\beta^{4} d \rho d \beta d \gamma d \Omega$ where $d \Omega=\sin \theta_{2} d \theta_{1} d \theta_{2} d \theta_{3}$ is the volume element of the Euler angles.

In order to obtain approximate solutions for the dynamics, we expand the potential energy arround the axial minimum according to eq. (48). Near the minimum, the moments of inertia are given by :

$$
\mathcal{J}_{1}=\mathcal{J}_{2} \simeq \bar{\rho}^{2},
$$

and

$$
\mathcal{J}_{3}=\frac{2 \bar{\rho}^{2}}{\bar{\epsilon}} \eta^{2}
$$


This yields for the kinetic rotational energy :

$$
T_{\text {rot }}=\frac{1}{L^{3}}\left(\frac{L^{2}-L_{3}^{2}}{2 \bar{\rho}^{2}}+\frac{\bar{\epsilon}}{4 \bar{\rho}^{2}} \frac{L_{3}^{2}}{\eta^{2}}\right) .
$$

The last term in $T_{\text {rot }}$ is a rotation-vibration interaction. The presence of this centrifugal barrier has a deep connection with the gauge invariance. It arises because when two $\lambda$ 's are equal we can not define the rotation angles in the polar representation for $A_{i a}$. As a consequence, there is in this case no dynamics associated to these degrees of freedom.

By keeping $\mathcal{J}_{1}$ and $\mathcal{J}_{2}$ constants, we will neglect further rotation-vibration interaction terms. For small angular momentum, we can treat them in perturbation. We have checked that they are indeed small, which is expected since the deformation is large.

The eigenfunctions of $\mathrm{H}$ are of the form [10] :

$$
\begin{aligned}
\varphi\left(\delta \rho, \delta \epsilon, \eta, \theta_{i}\right)=\left(\frac{2 I+1}{16 \pi^{2}\left(1+\delta_{K 0}\right)}\right)^{1 / 2} & R_{n_{\rho}}(\delta \rho) g_{n_{\beta}}(\delta \epsilon) \chi_{n_{\gamma}}^{K}(\eta) \\
& \times\left(D_{M K}^{I *}\left(\theta_{i}\right)+(-1)^{I} D_{M-K}^{I *}\left(\theta_{i}\right)\right),
\end{aligned}
$$

where $R_{n_{\rho}}$ are the solutions of the harmonic oscillator of frequence $\omega_{\rho}$. For the functions $g_{n_{\beta}}(\delta \epsilon)$ and $\chi_{n_{\gamma}}^{K}(\eta)$, we obtain the following expressions :

$$
g_{n_{\beta}}(\delta \epsilon)=A \exp \left(-\frac{1}{4 \bar{\epsilon}^{2}}(\delta \epsilon+\bar{\epsilon})^{2}\right) H_{n_{\beta}}\left(\frac{\delta \epsilon}{\sqrt{2} \bar{\epsilon}}\right)
$$

and

$$
\chi_{n_{\gamma}}^{K}(\eta)=B|\eta|^{1 / 2} \eta^{K / 2} \exp \left(-\frac{\lambda}{2} \eta^{2}\right){ }_{1} F_{1}\left(-n_{\gamma}, \frac{|K|}{2}+\frac{1}{2}, \lambda \eta^{2}\right),
$$

where $H_{n_{\beta}}$ are the Hermite polynomials, ${ }_{1} F_{1}$ is the hypergeometric function and $\lambda=3 / 2 \bar{\epsilon}^{2}$. A and $\mathrm{B}$ are normalization constants.

We thus see from eq. (61) that dynamical effects arising from the collective kinetic energy shift the center of the gaussian away from the minimum of the potential energy at $\beta=1-\bar{\epsilon}$ to the point $\beta=1$. This point was already known to be the minimum of the magnetic energy eq. (27). However 
an additional information we have gained from eq. (35) is that the boundary condition on the wave function is precisely that it must vanish at $\beta=1$. This implies that only odd values of $n_{\beta}$ are acceptable. A second boundary condition on the wave function is that it vanishes at $\eta^{2}=\bar{\epsilon}^{2} / 3$. For $\beta$ near 1 and $\gamma$ small, we have from eq. (18) and eq. (28) : $\left(\lambda_{1} \lambda_{2} \lambda_{3}\right)^{2}=\bar{\rho}^{6}\left(\bar{\epsilon}^{2}-3 \eta^{2}\right) / 9$. From the expression for $\chi_{n_{\gamma}}^{K}(\eta)$, we see that the wave function is indeed negligible at the boundary $\eta^{2} \simeq \bar{\epsilon}^{2} / 3$.

The functions (60) describe states of positive parity, $\mathrm{K}$ being restricted to positive even integers and $I=0,1,2$ for $K=0$ and $I=K, K+1 \ldots$ for $K \neq 0$.

The eigenvalues of the energy are given by :

$$
\begin{aligned}
E_{I K n_{\rho} n_{\beta} n_{\gamma}}= & \left(n_{\rho}+\frac{1}{2}\right) \frac{1}{L} \omega_{\rho}+\left(n_{\beta}+\frac{3}{4}\right) E_{\beta}+\left(2 n_{\gamma}+\frac{1}{2}|K|+1\right) E_{\gamma} \\
& +\frac{1}{2 L^{2} \bar{\rho}^{2}}\left(I(I+1)-K^{2}\right) \frac{1}{L}+\bar{V}^{\prime}
\end{aligned}
$$

where

$$
E_{\beta}=E_{\gamma}=\frac{3}{L}\left(\frac{2}{3}\right)^{2 / 3} g^{2 / 3} \frac{1}{\bar{\epsilon}^{1 / 3}},
$$

and $\omega_{\rho}$ is given by (45).

The $\rho$ vibration is therefore softer than the $\beta$ and $\gamma$ vibrations. Note that the zero point vibrational energy for the $\beta$ vibration is $7 / 4 E_{\beta}$ instead of the usual value $1 / 2$.

In the strong coupling approximation, the energy is proportional to $g^{2 / 3}$. By using $\bar{\epsilon}=2 / 33$, one finds :

$$
\omega_{\rho}=1.24 g^{2 / 3}
$$

and

$$
E_{\beta}=E_{\gamma}=5.83 g^{2 / 3} / L
$$

The lowest excited energy levels are the states of the ground state rotational band $K=0: m\left(2^{+}\right)=0.35$ and $m\left(4^{+}\right)=1.18$, the states of the 
$\rho$-vibrational band $n_{\rho}=1: m\left(0^{+}\right)=1.24, m\left(2^{+}\right)=1.59$ and the state in the band $n_{\rho}=2: m\left(0^{+}\right)=2.5$ (all the masses are given in units of $1 / L$ ).

For the $\rho$ vibration, we have also investigated the importance of the anharmonic terms. At the lowest order in $\bar{\epsilon}$, the collective potential is given by

$$
V^{\prime}(\rho)=g \rho+\frac{99}{8 L^{3} \rho^{2}},
$$

or, using the appropriate rescaling $\rho=g^{1 / 3} \tilde{\rho} / L$,

$$
V^{\prime}(\tilde{\rho})=g^{2 / 3}\left(\tilde{\rho}+\frac{99}{8 \tilde{\rho}^{2}}\right) \frac{1}{L} .
$$

The cubic and quartic terms give sizeable contributions of opposite signs. If we approximate the collective potential by a quartic polynomial, the mass of the first $0^{+}$excited state is lowered to : $m\left(0^{+}\right) \simeq 1.0 / L$.

To perform a comparison with experimental data it would be necessary to generalize the present calculation to the $\mathrm{SU}(3)$ group and to include dynamical quarks. The experimental situation however appears somewhat unsettled [13].

\section{$5 \quad$ Discussion and perspectives}

In order to compare with the approaches which consider gauge invariant variables constructed from the magnetic field [1, 11], it is usefull to remember the following relations valid in the strong coupling limit between the three eigenvalues $b_{n}^{2}$ of the matrix $B_{i a} B_{j a}$ and the three $\lambda$ 's :

$$
b_{1}=g \lambda_{2} \lambda_{3}, b_{2}=g \lambda_{1} \lambda_{3}, b_{3}=g \lambda_{1} \lambda_{2} .
$$

We have also B.y $=6 b_{1} b_{2} b_{3}$.

Our result for the behaviour of the wavefunction in the infrared region is in agreement with the conclusion of K. Johnson [1], who found that the vacuum wave function should vanish at $\operatorname{det} B=0$. The results $\bar{\rho}^{2} \neq 0$ and 
$B^{2} \neq 0$ at the minimum cannot be obtained in a perturbative approach. We have found a strongly deformed minimum and the lowest energy levels correspond to a rotational band and a $\rho$-vibrational band. Our predictions for the lowest glueball states are different from those of Lüscher and Münster who have performed a perturbative calculation for the $S U(2)$ gauge theory in a finite volume [6]. They have choosen a basis of eigenfunctions proportional to $\exp \left(-\frac{1}{2} \omega A_{i a} A_{i a}\right)$ to diagonalize their effective hamiltonian. This basis is not adapted to describe our wave functions (60) : a very large number of terms would be necessary to have a sufficient accuracy. In contrast, if we had obtained a minimum located at $\bar{\rho}=0$, a gaussian in the cartesian coordinates would have been an acceptable ansatz [7]. Because the minimum is located at $\bar{\rho} \neq 0$, the correct ansatz is a gaussian in the "curvilinear coordinates" $\delta \rho, \delta \epsilon$ and $\eta$, which cannot be expressed as a gaussian in the cartesian coordinates $A_{i a}$.

Let us make some remarks about the derivative expansion. This is an expansion in powers of $1 / g^{2 / 3}$. It is expected to be valid when the derivatives of gauge invariant quantities are small compared to some scale, for instance $\left|\partial_{i} \lambda_{n}\right| \ll \bar{\rho}^{2}$. In lowest order in the strong coupling approximation, there is no propagation and the wavefunctional reduces to a function of gauge invariant quantities constant in space. The next step is to investigate the coupling between the sites and its effect on the vacuum state properties and the glueball spectrum.

For color singlet states of zero angular momentum, the first term involving derivatives in the kinetic energy is :

$$
T_{1}=-\frac{1}{2 g^{2}}\left\{\sum_{n, m, k} \lambda_{n} S_{n m}^{k}\left(\vec{\nabla} \cdot \vec{f}^{k}\right) \frac{\delta}{\delta \lambda_{k}} \lambda_{n} S_{n m}^{k}\left(\vec{\nabla} \cdot \vec{f}^{k}\right) \frac{\delta}{\delta \lambda_{k}}\right\},
$$

where

$$
S_{n m}^{k}=-\frac{\epsilon_{k n m}}{\lambda_{n}^{2}-\lambda_{m}^{2}}
$$


and $\vec{f}^{n}$ are the column vectors of the matrix $\hat{f}$. For the potential energy, the terms containing derivatives are exactly given by the last term in eq. (9). In order to check the accuracy of our variational Ansatz, it will be usefull to compare our results with other $\mathrm{SU}(2)$ calculations : finite volume results [6], lattice Monte Carlo calculations [8] and analytical strong coupling expansions [9]. This comparison will also provide a usefull guide to construct variational Ansätze for negative parity states which are not included in our present variational space. Indeed the rescaling we have performed implies trial wave functions of the form $\Psi\left(\lambda_{1}^{2}, \lambda_{2}^{2}, \lambda_{3}^{2}\right)$.

Aknowledgements One of us (C. M.) thanks K. Johnson for very enlightening discussions.

\section{References}

[1] K. Johnson, in QCD-20 Years Later, Aachen, June 1992;

D. Z. Freedman, P. E. Haagensen, K. Johnson and J. I. Latorre, MIT preprint CTP\#2238, August 1993.

[2] Y. A. Simonov, Sov. J. Nucl. Phys. 41 (1985), 835.

[3] G. K. Savvidy, Phys. Lett. 159B (1985), 325.

[4] A. Bohr, Mat. Fys. Medd. Dan. Vid. Selsk. 26, No. 14 (1952).

A. Bohr and B. R. Mottelson, Nuclear Structure, Vol 2, Benjamin (1975).

[5] W. Zickendraft, Journal of Mathematical Physics, 12 (1971), 1663.

[6] M. Lüscher and G. Münster, Nucl. Phys. B232 (1984), 445;

J. Koller and P. van Baal, Nucl. Phys. B302 (1988), 1.

[7] A. K. Kerman and D. Vautherin, Ann. of Phys. (N.Y.) 192 (1989), 408. 
[8] B. A. Berg, A. Billoire and C. Vohwinkel, Phys. Rev. Lett. 57 (1986), 400;

C. Michael and M. Tepper, Nucl. Phys. 305 (1988), 453; Lattice 93 Nucl. Phys. (Proc. Suppl.) 30 (1993).

[9] G. Münster, Nucl. Phys. 190 (1981) 439.

[10] J. M. Eisenberg and W. Greiner, Nuclear Models, North-Holland, Amsterdam (1970), chap. 5.

[11] S. Levit, "Body Fixed Frame, Rigid Gauge Rotations and Large N Random Fields in QCD”, WIS-preprint \# 93140.

[12] A. M. Badalyan, Sov. J. Nucl. Phys. 38 (1983), 464.

[13] M. Burchell, Physics in collision 12, edited by J. Cumalat, Editions Frontieres (1993). 